\title{
Critical Behavior of a Heavy Particle in a Granular Fluid
}

\author{
Andrés Santos and James W. Dufty \\ Department of Physics, University of Florida, Gainesville, FL 32611
}

(October 28, 2018)

\begin{abstract}
Behavior analogous to a second order phase transition is observed for the homogeneous cooling state of a heavy impurity particle in a granular fluid. The order parameter $\phi$ is the ratio of impurity mean square velocity to that of the fluid, with a conjugate field $h$ proportional to the mass ratio. A parameter $\beta$, measuring the fluid cooling rate relative to the impurity-fluid collision rate, is the analogue of the inverse temperature. For $\beta<1$ the fluid is "normal" with $\phi=0$ at $h=0$, as in the case of a system with elastic collisions. For $\beta>1$ an "ordered" state with $\phi \neq 0$ occurs at $h=0$, representing an extreme breakdown of equipartition. Critical slowing and qualitative changes in the velocity distribution function for the impurity particle near the transition are noted.
\end{abstract}

PACS number(s): 45.70.-n, 45.70.Mg, 05.20.Dd

The simplest statistical mechanical model for an activated granular fluid is a system of smooth, inelastic hard spheres. The inelasticity is specified in terms of a restitution coefficient $\alpha \leq 1$. Qualitative differences between the cases $\alpha=1$ (elastic spheres) and $\alpha \neq 1$ have been demonstrated in many ways using both theoretical and simulation methods [1]. Among these is the replacement of the Gibbs state for an isolated uniform system by a homogeneous cooling state (HCS). The HCS is inherently time dependent due to the loss of energy in each binary collision. It is postulated that the time dependence of this ensemble occurs entirely through a scaling of the velocity by its root mean square ("thermal") velocity $v_{T}(t)$ and the associated normalization [2]. In this case, the dynamics (Liouville equation or kinetic equation) can be transformed to a stationary state form using suitable dimensionless variables. The time dependence of $v_{T}(t)$ also follows from this scaling, so that only a time independent dimensionless cooling rate must be determined selfconsistently. Similar results apply for a multi-component system, although the analysis is complicated by the existence of many scaling velocities [3]. The HCS results when the cooling rates for all mean square velocities are the same. This condition then determines all scaling velocities in terms of that for one species. It is the analogue of energy equipartition for a system with $\alpha=1$, but deviations from equipartition occur for all $\alpha \neq 1$. These deviations can be weak or strong depending on the mechanical differences between the species and the degree of dissipation.

Many of the new effects to be expected in multicomponent systems can be illustrated by the simplest case of a single impurity particle in a one component system [ 4 . The fluid (impurity) particle diameter, mass, and restitution coefficient are denoted by $\sigma\left(\sigma_{0}\right), m\left(m_{0}\right)$, and $\alpha\left(\alpha_{0}\right)$. There are two mean square velocities and associated cooling rates for the fluid and impurity particles,

$$
\begin{aligned}
& v_{T}^{2}(t)=\frac{2}{3}\left\langle v^{2}(t)\right\rangle, \quad v_{T 0}^{2}(t)=\frac{2}{3}\left\langle v_{0}^{2}(t)\right\rangle, \\
& \xi=-\partial_{t} \ln v_{T}^{2}(t), \quad \xi_{0}=-\partial_{t} \ln v_{T 0}^{2}(t) .
\end{aligned}
$$

The brackets denote an average over the HCS, and the subscript 0 denotes properties of the impurity particle. The cooling rates for a moderately dense fluid can be calculated to good accuracy from the Boltzmann-Enskog and Lorentz-Enskog kinetic equations for the fluid and impurity reduced distribution functions [3]. These rates then depend on the velocity distributions for the fluid and impurity particle in the HCS and can be estimated to good approximation using Maxwellians (see justification below) with the results

$$
\begin{gathered}
\xi^{*}=\frac{\xi}{n \sigma^{2} g v_{T}}=\frac{2 \sqrt{2 \pi}}{3}\left(1-\alpha^{2}\right), \\
\xi_{0}^{*}=\frac{\xi_{0}}{n \sigma^{2} g v_{T}}=\nu^{*}(1+\phi)^{1 / 2}\left(1-h \frac{1+\phi}{\phi}\right),
\end{gathered}
$$

where $n$ is the fluid density, $g$ is the pair correlation function for two fluid particles, and $n \sigma^{2} g v_{T}$ is a characteristic collision rate for the fluid particles. Similarly, $\nu^{*}=\nu / n \sigma^{2} g v_{T}=(16 \sqrt{\pi} / 3) h\left(g_{0} / g\right)(\bar{\sigma} / \sigma)^{2}$ is a corresponding dimensionless average impurity-fluid particle collision rate, where $\bar{\sigma}=\left(\sigma+\sigma_{0}\right) / 2$ and $g_{0}$ is the corresponding fluid-impurity particle pair correlation function. The dependence on the mass ratio occurs entirely through $h=m\left(1+\alpha_{0}\right) / 2\left(m+m_{0}\right)$. The time independent ratio of mean square velocities,

$$
\phi=v_{T 0}^{2} / v_{T}^{2},
$$

is determined by the condition that the cooling rates be equal,

$$
h=\frac{\phi}{(1+\phi)^{3 / 2}}\left[(1+\phi)^{1 / 2}-\beta\right] .
$$

The constant $\beta$ is a measure of the fluid cooling rate relative to the fluid-impurity collision rate, 


$$
\beta=\frac{\xi^{*}}{\nu^{*}}=\frac{\left(1-\alpha^{2}\right)}{4 \sqrt{2} h} \frac{g}{g_{0}}\left(\frac{\sigma}{\bar{\sigma}}\right)^{2} .
$$

The elastic collisions limit is given by $\beta=0$, for which the solution is $\phi=h /(1-h)=m / m_{0}$. This is the required result from the equipartition theorem. If only the fluid particle collisions are elastic $\left(\beta=0, \alpha_{0} \neq 1\right)$, a recent exact result of Martin and Piasecki, $\phi=m(1+$ $\left.\alpha_{0}\right) /\left[2 m_{0}+m\left(1-\alpha_{0}\right)\right]$ is recovered [5]. More generally, Eq. (5) determines $\phi(\beta, h)$ for the entire physical domain of $h$ and $\beta$. The solution is found to have qualitatively different behavior at small $h$ for different values of $\beta$. The asymptotic behavior for $h \rightarrow 0$ is found to be

$$
\phi(\beta, h) \rightarrow \begin{cases}(1-\beta)^{-1} h, & \beta<1 \\ \sqrt{2 h}, & \beta=1 \\ \beta^{2}-1+2 \beta^{4}\left(\beta^{2}-1\right)^{-1} h, & \beta>1\end{cases}
$$

Thus $\phi$ vanishes at $h=0$ for $\beta<1$ but is finite at $\beta>1$. The condition $\beta<1$ is "normal" in the sense that $\phi$ scales as the mass ratio just as for systems with elastic collisions and the consequent equipartition of kinetic energy. This normal state has been studied recently for $h \ll 1$, where the kinetic theory implies a corresponding Fokker-Planck description [4]. The condition $\beta>1$ corresponds to an extreme violation of equipartition such that the mean square velocities of the fluid and impurity particles remain comparable even though their mass ratio is zero. Figure 1 shows the more complete dependence of $\phi(\beta, h)$ on $h$ for $\beta=0.9,1$, and 1.1, confirming the above asymptotic analysis.

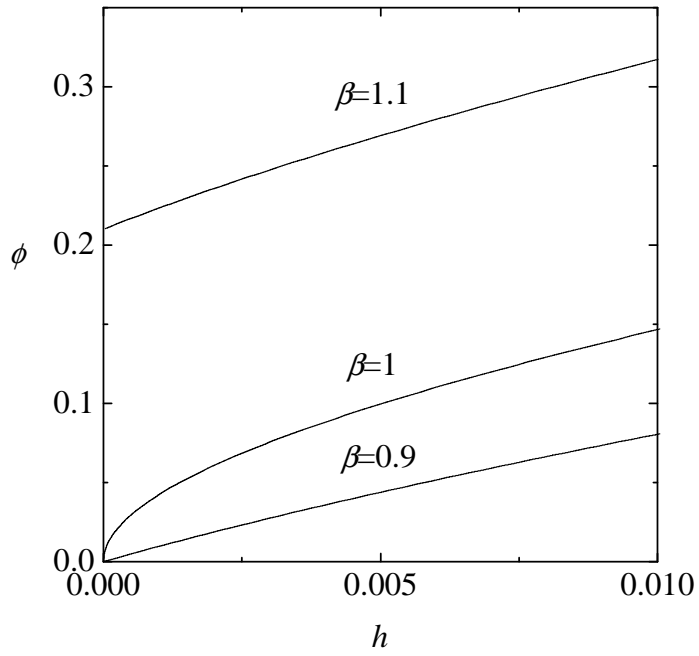

FIG. 1. Ratio of mean square velocities, $\phi$, as a function of the mass ratio parameter $h$ for $\beta=0.9,1$, and 1.1.

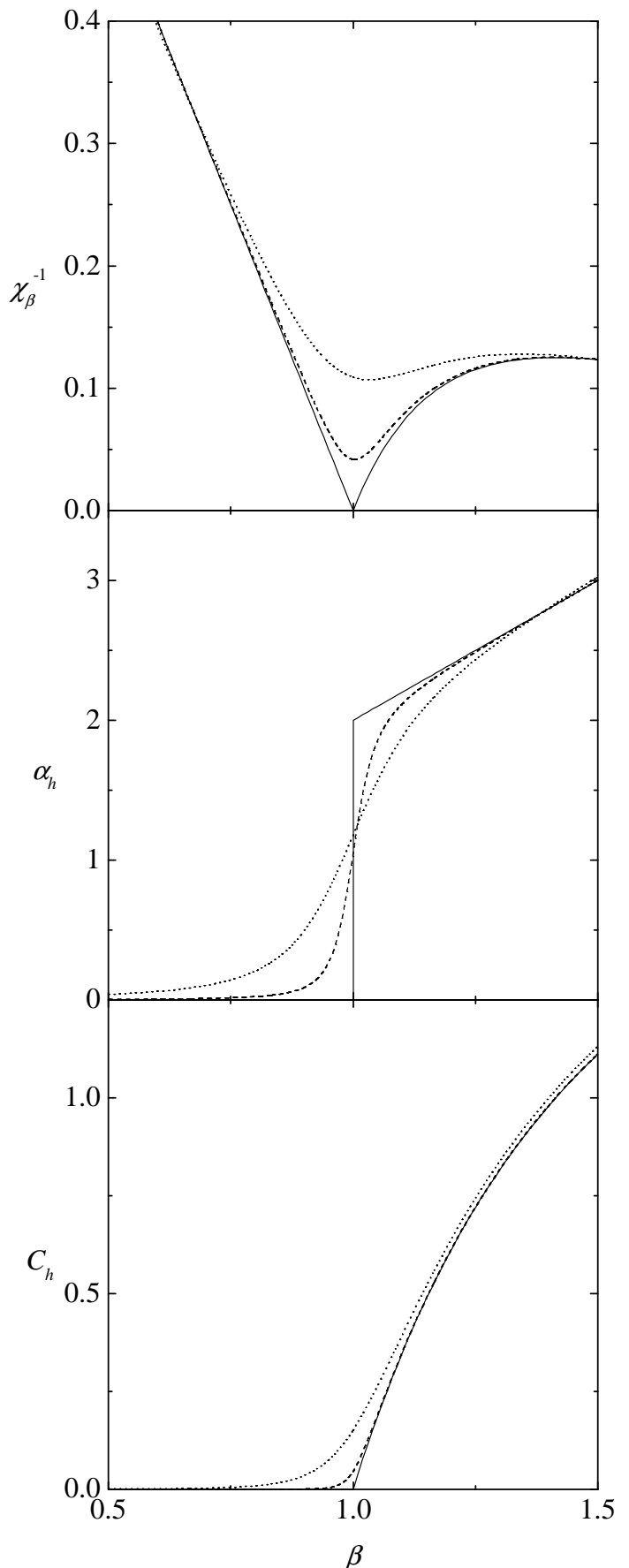

FIG. 2. Inverse susceptibility $\left(\chi_{\beta}^{-1}\right)$, expansion coefficient $\left(\alpha_{h}\right)$, and heat capacity $\left(C_{h}\right)$ as functions of $\beta$ for $h=10^{-2}$ (dotted line), $h=10^{-3}$ (dashed line), and $h=0$ (solid line).

This behavior at small $h$ is analogous to a thermodynamic transition at $\beta=1$ between two different states of the system characterized by the order parameter $\phi$, conjugate field $h$, and inverse "temperature" $\beta$. To develop this analogy a Helmholtz free energy $F(\beta, \phi)$ is obtained from the "equation of state" (5) by integrating the definition $h=\partial F(\beta, \phi) / \partial \phi$ [with the boundary condition $F(\beta, 0)=0$, for simplicity]. Next, the Gibbs free energy is constructed from $F$ by the Legendre transfor- 
mation $\Phi(\beta, h)=F-h \phi$. The first and second derivatives of $\Phi(\beta, h)$ provide the order parameter $\phi$, "entropy" $\Sigma$, "susceptibility" $\chi_{\beta}$, "expansion coefficient" $\alpha_{h}$, and "heat capacity" $C_{h}$. The results are

$$
\begin{gathered}
\Phi(\beta, h)=(1-h) \phi-\ln (1+\phi)-2 \beta\left[\frac{2+\phi}{(1+\phi)^{1 / 2}}\right. \\
\Sigma(\beta, h)=\frac{\partial \Phi(\beta, h)}{\partial \beta}=-2\left[\frac{2+\phi}{(1+\phi)^{1 / 2}}-2\right], \\
\chi_{\beta}=-\frac{\partial^{2} \Phi(\beta, h)}{\partial h^{2}}=\frac{(1+\phi)^{5 / 2}}{(1+\phi)^{1 / 2}-\beta\left(1-\frac{1}{2} \phi\right)}, \\
\alpha_{h}=-\frac{\partial^{2} \Phi(\beta, h)}{\partial \beta \partial h}=\chi_{\beta} \frac{\phi}{(1+\phi)^{3 / 2}}, \\
C_{h}=-\frac{\partial^{2} \Phi(\beta, h)}{\partial \beta^{2}}=\chi_{\beta}^{-1} \alpha_{h}^{2} .
\end{gathered}
$$

The behavior of these functions near $h=0$ follows directly from (7). For example, the response functions $\chi_{\beta}$, $\alpha_{h}$, and $C_{h}$ at $h=0,10^{-2}$, and $10^{-3}$ are shown in Fig. 2. In the limit $h \rightarrow 0$, the entropy and order parameter are continuous at $\beta=1$, so the divergence of $\chi_{\beta}$ and the discontinuity of $\alpha_{h}$ at $\beta=1$ identifies this as a second order phase transition. Near the critical region ( $h \ll 1,|\beta-1| \ll 1)$, the free energy adopts the Landaulike form $\Phi(\beta, h) \approx \frac{1}{2}(1-\beta) \phi^{2}+\frac{1}{6} \phi^{3}-h \phi$, which yields $h \approx(1-\beta) \phi+\frac{1}{2} \phi^{2}$. As a consequence, the free energy and the equation of state in the critical region satisfy the scaling relations $\Phi\left(\lambda(\beta-1), \lambda^{a} h\right)=\lambda^{b} \Phi(\beta-1, h)$ and $\phi\left(\lambda(\beta-1), \lambda^{a} h\right)=\lambda^{b-a} \phi(\beta-1, h)$, with $a=2$ and $b=3$. These scaling relations suffice to determine the critical exponents [6] $\hat{\delta}=a /(b-a)=2, \hat{\beta}=b-a=1$, and $\hat{\gamma}=2 a-b=1$, while the critical exponent $\hat{\alpha}=2-b=-1$ is negative, indicating that $C_{h}$ is continuous at the critical point.

If the ratio between the initial mean square velocities of the fluid and impurity particles is not that given by the solution to (5), there is an evolution to the HCS described by (1) which can be written in the Ginzburg-Landau form

$$
\partial_{s} \phi=-\left[\xi_{0}^{*}(t)-\xi^{*}(t)\right] \phi=-\mu(\phi) \frac{\partial \Phi(\beta, h ; \phi)}{\partial \phi},
$$

where a new time scale $d s=n \sigma^{2} g v_{T}(t) d t$ has been introduced. Here, $\Phi(\beta, h ; \phi)$ is a variational free energy given by Eq. (8) with the order parameter $\phi$ considered as an independent variable, and the kinetic coefficient $\mu(\phi)$ is

$$
\mu(\phi)=\nu^{*}(1+\phi)^{3 / 2}
$$

The stationary solution occurs for $\partial \Phi(\beta, h ; \phi) / \partial \phi=0$, which is just Eq. (5). For states near the HCS the equation can be linearized and a characteristic response time $\tau$ identified according to

$$
-\partial_{s} \ln \left(\phi-\phi_{\mathrm{HCS}}\right)=\tau^{-1}=\left(\mu \chi_{\beta}^{-1}\right)_{\phi_{\mathrm{HCS}}}
$$

where $\phi_{\mathrm{HCS}}$ denotes the solution to (5). In the elastic limit $\tau$ is just the equilibration time for the impurity particle to attain a mean kinetic energy equal to that of the fluid particles. Similarly, for inelastic collisions it is the time (in units of the fluid mean free time) for the impurity particle to reach a cooling rate equal to that of the fluid. This characteristic time is a smooth function of $h$ and $\beta$ except in the limit $h \rightarrow 0$ where $\tau$ diverges at $\beta=1$. This critical slowing follows directly from the fact that $\tau \propto \chi_{\beta}$. Otherwise, the relaxation times away from $\beta=1$ are finite and comparable for the normal and ordered states. Figure 3 shows the dependence of the inverse time $\tau^{*-1} \equiv\left(\nu^{*} \tau\right)^{-1}$ on $h$ for $\beta=0.9,1$, and 1.1. The quantity $\tau^{*}$ measures the relaxation time for the impurity particle in terms of the number of impurityfluid particle collisions.

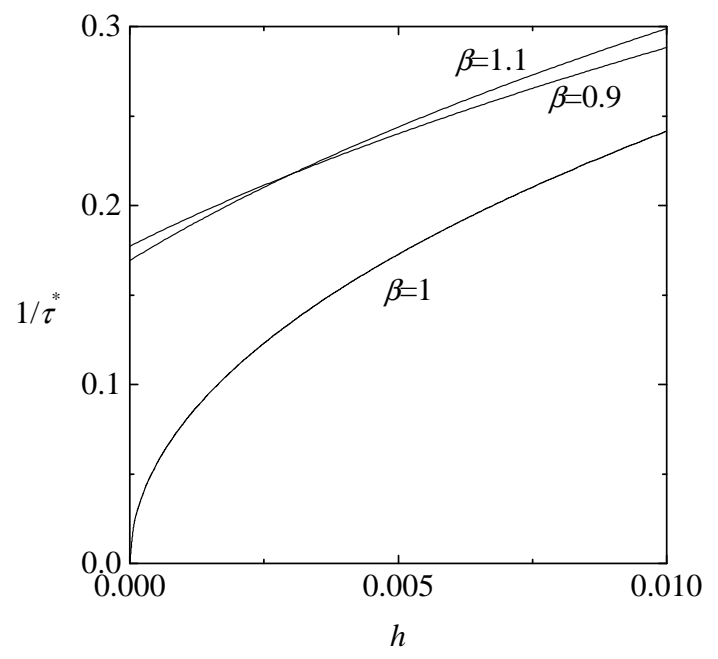

FIG. 3. Inverse characteristic time $\tau^{*-1} \equiv\left(\nu^{*} \tau\right)^{-1}$ as a function of the mass ratio parameter $h$ for $\beta=0.9,1$, and 1.1 .

The above analysis is based on an approximate evaluation of the cooling rates using Maxwellians for the impurity and fluid particle velocity distributions. This is known to be accurate for the fluid particles [2] but the new features in the critical domain and in the ordered phase $\beta>1$ require closer inspection of the impurity particle distribution. Based on the Lorentz-Enskog kinetic equation, it is possible to determine the impurity particle velocity distribution for the HCS asymptotically close to $\beta \rightarrow 1$ and $h \rightarrow 0$ with the result

$$
F\left(\mathbf{v}^{*}\right) \rightarrow C \exp \left\{-\frac{\phi}{h}\left[(1-\beta) v^{* 2}+\frac{1}{10} \phi v^{* 4}\right]\right\}
$$


where $\mathbf{v}^{*}=\mathbf{v}_{0} / v_{T 0}$ and $C$ is a normalization constant. The asymptotic equation of state is determined from $\int d \mathbf{v}^{*}\left(v^{* 2}-\frac{3}{2}\right) F\left(\mathbf{v}^{*}\right)=0$. The equation of state is similar to that of Eq. (7) with only minor quantitative changes in some coefficients. This justifies the preceding analysis based on the Maxwellian approximation to $\xi_{0}^{*}$. Nevertheless, the distribution function itself shows new qualitative differences. As expected, for $\beta<1, \phi \rightarrow$ $h /(1-\beta)$ and the Maxwellian is recovered. For $\beta>1$, $\phi \rightarrow 10(\beta-1) / 3$ and the velocity distribution becomes sharp at $v^{*}=\sqrt{3 / 2}, F\left(\mathbf{v}^{*}\right) \rightarrow(6 \pi)^{-1} \delta\left(v^{*}-\sqrt{3 / 2}\right)$. Furthermore, at the critical point $(\beta=1), \phi \rightarrow \sqrt{10 \lambda h}$ and $\left.F\left(\mathbf{v}^{*}\right)\right) \rightarrow C \exp \left(-\lambda v^{* 4}\right)$, where $\lambda \simeq 0.24$. This crossover from Gaussian to exponential quartic to delta distributions is illustrated in Fig. 4 for $h=10^{-2}$ and $\beta=0.9,1.0$, and 1.1 .

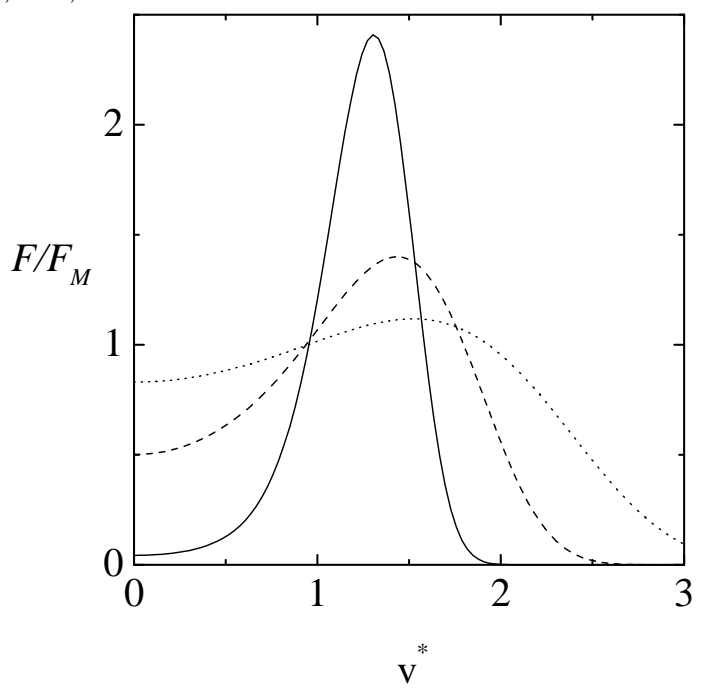

FIG. 4. Velocity distribution function of the impurity particle, $F$, relative to the Maxwellian, $F_{M}$, for $h=10^{-2}$ and $\beta=0.9$ (dotted line), $\beta=1$ (dashed line), and $\beta=1.1$ (solid line).

The origin of this transition can be traced to the fact that the cooling of the fluid is due to collisions that depend on the restitution coefficient $\alpha$ but not the mass ratio (or $h$ ), while cooling of the impurity depends on the mass ratio but not $\alpha$. The equality of cooling rates is enforced by adjusting the ratio of the mean square velocities, $\phi$. In general, for fixed mechanical properties, this is possible for small mass ratio only if $\phi$ is non zero (the ordered state); the cooling rate for the impurity has an explicit proportionality to $h$ which must be countered by $\phi$. This implies that the mean kinetic energy of the impurity particle diverges relative to that of the fluid particles. However, if this explicit decrease of the impurity cooling rate with $h$ is tempered by a large size ratio and/or weak fluid particle dissipation then the normal behavior with $\phi=0$ is restored. The parameter $\beta$ quantifies the meaning of "tempered". To give an explicit example, consider the case $\alpha=\alpha_{0}=0.95$ and $m / m_{0}=10^{-2}$. Also, for simplicity consider low density so that $g=g_{0} \rightarrow 1$. Then $\beta=1.79(\sigma / \bar{\sigma})^{2}$ and the system is close to the ordered state for $\sigma=\sigma_{0}$ but close to the normal state for $\sigma_{0}=2 \sigma$. It is possible to study the crossover through the critical domain using Direct Monte Carlo simulation of the Boltzmann-Enskog and Lorentz-Enskog kinetic equations, and by molecular dynamics simulation. In fact both Monte Carlo molecular dynamics simulations for the domain $\beta<1$ and $m / m_{0} \approx 10^{-2}$ have been performed to confirm Brownian motion in the normal state [7]. Extension of these studies to the critical and ordered states should be straightforward.

For experimental purposes it is perhaps more relevant to study these critical properties as reflected in the diffusion coefficient $D$ (or mean square displacement) for the impurity particle. The dependence of $D$ on $\beta$ and $h$ can be calculated from the Lorentz-Enskog kinetic equation in the same approximation as the cooling rates above. The studies of the Fokker-Planck limit $(h \rightarrow 0)$ in [4] suggest a divergence of $D$ as $\beta \rightarrow 1$ although that analysis is strictly limited to $\beta<1$. A more complete description of $D$ for all $\beta, h$ will be given elsewhere [8].

This research was supported by National Science Foundation grant PHY 9722133. A.S. acknowledges partial support from the DGES (Spain) through grant No. PB971501 and through a sabbatical grant No. PR2000-0117.

* Permanent address: Departamento de Física, Universidad de Extremadura, E-06071 Badajoz, Spain; Email address: andres@unex.es

† Email address: dufty@phys.ufl.edu

[1] See, for instance, H. M. Jaeger, S. R. Nagel, and R. P. Behringer, Rev. Mod. Phys. 68, 1259 (1996).

[2] J. J. Brey, M. J. Ruiz-Montero, and D. Cubero, Phys. Rev. E 54, 3664 (1996); T. P. C. van Noije and M. H. Ernst, Granular Matter 1, 57 (1998).

87, 1051 (1997).

[3] V. Garzó and J. W. Dufty, Phys. Rev. E 60, 5706 (1999).

[4] J. J. Brey, J. W. Dufty, and A. Santos, J. Stat. Phys. 97, 2811 (1999).

[5] P. A. Martin and J. Piasecki, Europhys. Lett. 46, 613 (1999).

[6] Following the thermodynamic analogy, the critical exponents $\hat{\delta}, \hat{\beta}, \hat{\gamma}$, and $\hat{\alpha}$ are defined as $\phi(\beta=1, h) \sim h^{1 / \hat{\delta}}$, $\phi(\beta>1, h=0) \sim(\beta-1)^{\hat{\beta}}, \chi_{\beta}(\beta, h=0) \sim|\beta-1|^{-\hat{\gamma}}$, $C_{h}(\beta, h=0) \sim|\beta-1|^{-\hat{\alpha}}$.

[7] J. J. Brey, M. Ruiz-Montero, R. García-Rojo, and J. W. Dufty, Phys. Rev. E 60, 7174 (1999).

[8] A. Santos and J. W. Dufty (unpublished). 